\title{
Relationship between Occlusal Factors, Oral Parafunctions and Temporomandibular Disorders: A Case Control Study
}

\author{
Caroline Mélou ${ }^{1,3}$, Lucie Leroux ${ }^{2}$, Fleur Meary ${ }^{1,3}$, Valérie Bertaud ${ }^{1,4}$, Clément Le Padellec ${ }^{1}$, Arnaud Lemaire ${ }^{1}$ and Dominique \\ Chauvel-Lebret ${ }^{1,3, *}$
}

${ }^{1} \mathrm{CHU}$ Rennes, Pôle d'Odontologie, Rennes, France

${ }^{2} 1$ Place Jean Jaurès, Guéméné-Penfao, France

${ }^{3}$ Univ Rennes, CNRS, ISCR (Institut des Sciences Chimiques de Rennes) Rennes, France

${ }^{4}$ Univ Rennes, Inserm, LTSI (Laboratoire Traitement du Signal et de l'Image) Rennes, France

*Corresponding author: Dominique Chauvel-Lebret, Groupe Biomatériaux en Site Osseux, UMR CNRS 6226, Université de Rennes 1, 2 avenue du Pr Léon Bernard, Bâtiment 15, 35043 Rennes Cedex, France, Tel: 33223234364; E-mail: dominique.lebret-chauvel@univ-rennes1.fr

Received: 29 Apr, 2019 | Accepted: 13 May, 2019 | Published: 17 May, 2019

Citation: Mélou C, Leroux L, Meary F, Bertaud V, Le Padellec C, et al. (2019) Relationship between Occlusal Factors, Oral Parafunctions and Temporomandibular Disorders: A Case Control Study. Int J Dent Oral Health 5(4): dx.doi.org/10.16966/2378-7090.295

Copyright: (C) 2019 Mélou C, et al. This is an open-access article distributed under the terms of the Creative Commons Attribution License, which permits unrestricted use, distribution, and reproduction in any medium, provided the original author and source are credited.

\section{Abstract}

Aims: The purpose of this study was to assess the multifactorial etiology of temporomandibular disorders (TMD). To this end, prevalence of occlusal factors and parafunctional habits was investigated and correlated in two groups: patients with TMD (case group) and patients without TMD (control group).

Methods: Prevalence of 7 static and 4 dynamic malocclusion features, and some parafunctional activities (nail biting, teeth grinding, teeth clenching, lips and cheeks biting, pens chewing, mandibular jaw moves habit...) were recorded in the two groups. Case group consisted of 30 patients with a diagnosis of myofascial pain, with or without limited opening, and/or disk displacement with or without reduction. Control group consisted of 30 patients without TMD. Univariate and multivariate analysis were used to identify the significant associations between occlusal variables, oral parafunctions and temporomandibular disorders.

Results: All patients in case group, and $93.3 \%$ in control group had at least one malocclusion, and a statistically significant association between TMD and three malocclusions (two dynamic malocclusion features: mediotrusive interferences, laterotrusive interferences, and one static malocclusion: overbite $>4 \mathrm{~mm}$ ) was shown. Regarding parafunctional activities, all patients in case group, and 93,3\% in control group had at least one parafunction, and the mean number of parafunctions per patient was significantly higher in case group than in control group. Besides, an association between TMD and some parafunctions was shown.

Conclusion: Given the multifactorial nature of TMD, a behavioral reeducation to reduce parafunctional activities could be the first phase of the care for TMD patients and could prevent TMD development. Besides, special attention should be given to normocclusion criteria. These criteria must be fulfilled for any dental care.

Keywords: Malocclusion; Occlusal factors; Orofacial pain; Parafunctional habits; Temporomandibular disorders

\section{Introduction}

Temporomandibular Disorders (TMD) are a heterogeneous group of conditions affecting temporomandibular joint, masticatory muscles and the near structures [1]. TMD result from association of biological, environmental, social and cognitive factors, and emotional behaviors [2]. These factors are classified as predisposing (structural, metabolic and/or psychological conditions), initiating (trauma or repetitive adverse loading of the masticatory system) and aggravating (parafunctional, hormonal or psychosocial factors) [3].

The literature shows that TMD have a multifactorial etiology [4]. For example, oral parafunctions might increase the risk of developing
TMD [5-8]. The role of dental occlusion stays controversial. If dental occlusion cannot be the dominant cause of TMD, it also cannot be considered negligible [9-11]. Some authors say that the role of occlusion in the etiology of TMD has been diminished over the years, but malocclusions can decrease adaptative capacity of masticatory system and influence the overall resistance of musculoskeletal system [3,9,11]. Furthermore, subjects with TMD history seem to respond differently to malocclusion factors than patients without TMD history [12].

The feature of dental occlusion and parafunctional oral behaviors might be a risk factor for TMD $[5,6]$. To study the multifactorial etiology of TMD, occlusal factors and parafunctional habits, a case- 
control study was conducted in the Dental Clinic of the hospital of Rennes (France). The aim of this study was to compare prevalence of malocclusions and parafunctional activities between patient with TMD (case group) and patient without TMD (control group).

\section{Materials and Methods}

\section{Case study}

Subjects: 30 case patients were recruited in the TMD Department of the Dental Clinic of the hospital of Rennes (France). Patients consulting for the first time in this department (with no history of treatment for TMD) and diagnosed with TMD were included in this study.

Inclusion criteria were as follow: men and women over 18 years, absence of any previous or ongoing treatment for TMD, absence of rheumatologic disorders (such as fibromyalgia, rheumatoid polyarthritis, ankylosing spondylarthritis), absence of any other pain than orofacial and cervical pain, absence of important teeth loss, absence of complete denture wearing.

30 control patients without signs of TMD, seeking a dental checkup at the Dental Clinic, were recruited. The control patients were recruited if they satisfied the inclusion criteria and had no signs, symptoms or history of TMD.

All patients were included between October 2013 and July 2014. All patients signed a written informed consent before entering the study. The study protocol was approved by the Research Ethics Committee of Rennes University Hospital Center (Number 13-60).

Research protocol: Clinical examination for signs and symptoms of TMD was adapted from the Research Diagnostic Criteria for Temporomandibular Disorders (RDC/TMD) Axis I. Patients could be included in case group when they presented myofascial pain, with or without limited opening, and/or disk displacement with or without reduction.

For all patients, case and control, 11 occlusal variables were recorded during a clinical examination: posterior cross bite, overjet $>7 \mathrm{~mm}$, overbite $>4 \mathrm{~mm}$, anterior open bite, crowding of the lower incisors, missing and non-replaced posterior teeth, protrusive interferences, right and left mediotrusive interferences, right and left laterotrusive interferences, symmetric or asymmetric Retruded Contact Position (RCP), Angle's classification based upon the relationship between canines.

Identification of parafunctional habits was carried out by selfreports. Patients answered few questions about their oral behaviors: nail biting, teeth grinding, teeth clenching, lips and cheeks biting, pens chewing, mandibular jaw moves habit and all other parafunctions described by patients. Besides, the clinicians paid attention to these habits during clinical examination, in order to detect some parafunctions of which the patient would not be aware.

All anamnesis and clinical examinations were performed by the same two trained operators with expertise in TMD clinical assessment.

Statistical analysis: All variables were compared between groups using tests implemented in the EPI INFO 7.1 software, using a significance threshold of 0.05 . Quantitative variables were compared using a student test (or Mann-Whitney for small sample sizes), and qualitative variables were compared using a $\mathrm{Chi}^{2}$ test (or Yates, or Fisher test according to required sample size of each test). Logistic regression for the multivariate analysis of malocclusions, parafunctional activities and TMD symptoms was performed using SPSS Statistics 17.

\section{Results}

Case group consisted of 23 women and 7 men (mean age $36.8 \pm$ 13.8; range 18-69 years). Control group consisted of 14 women and 16 men (mean age $43.6 \pm 14.6$; range 21-75) (Table 1).

In case group, $80 \%$ had myofascial pain, with or without limited opening, $46.7 \%$ had disk displacement with or without reduction.

All patients in case group and $93.3 \%$ in control group had at least one malocclusion. However, the mean number of malocclusions per patient was higher in case group than in control group, but this difference wasn't significant ( $\mathrm{p}=0.054$, data not shown).

Regarding static malocclusions, prevalence of overbite $>4 \mathrm{~mm}$ was significantly higher in case group $(\mathrm{p}=0.038)$ than in control group (Table 2).

The occlusal variable "missing and non-replaced posterior teeth" had a significantly higher prevalence in control group $(\mathrm{p}=0.0084)$ (Table 2).

Regarding dynamic malocclusion features, prevalence of mediotrusive interferences $(\mathrm{p}=0.0122)$ and laterotrusive interferences $(p=0.0036)$ was significantly higher in case group than in control group (Table 3).

In multivariate analysis, the statistically significant associations between TMD and overbite $>4 \mathrm{~mm}(\mathrm{p}=0.030)$, and between TMD and interferences in laterotrusion $(\mathrm{p}<0.001)$ was confirmed (Table 4$)$.

Furthermore, the feature of normocclusion "Class I and overbite $\leq 4 \mathrm{~mm}$ " was significantly more present among control patients than among case patients, $(\mathrm{p}=0.02)$ (Table 5).

Regarding parafunctional activities, all patients in case group, and 93.3\% in control group had at least one parafunction, and the mean number of parafunctions per patient was significantly higher in case group than in control group ( $p<0,001$, data not shown). Three oral parafunctions were higher in case group than in control group: teeth clenching $(\mathrm{p}=0.02)$, lips and cheeks biting $(\mathrm{p}=0.009)$, mandibular jaw moves habit $(\mathrm{p}=0.0099)$ were statistically associated with TMD (Table 6).

To check for possible confounding factors, we took into account other possible etiologies with a multivariate analysis which included age, sex and malocclusions. Multivariate analysis confirmed the association between TMD, interferences in laterotrusion $(\mathrm{p}=0.002)$, and overbite $>4 \mathrm{~mm}(\mathrm{p}=0.028)$ (Table 7).

\section{Discussion and Conclusion}

Case group composed of $76.7 \%$ women, with a mean age of 36.8 years, was in agreement with epidemiological data of TMD $[3,7]$. Manfredini, et al. [1] noticed the absence of general-population literature data on the prevalence of dynamic malocclusion. Few studies

Table 1: Age and sex distribution of the two groups. (SD: Standard Deviation) $\left({ }^{*} \mathrm{p}<0.05, * * \mathrm{p}<0.005\right)$

\begin{tabular}{|c|c|c|c|c|}
\hline & $\begin{array}{l}\text { Case group } \\
(n=30)\end{array}$ & $\begin{array}{c}\text { Control group } \\
(n=30)\end{array}$ & Test & p-value \\
\hline Age-Mean \pm SD & $36.8 \pm 13.8$ & $43.6 \pm 14.6$ & Student & 0.069 \\
\hline Range in Years & $18-69$ & $21-75$ & & \\
\hline \multicolumn{5}{|l|}{ Gender (\%) } \\
\hline Male & $7(23.3 \%)$ & $16(53.3 \%)$ & \multirow{2}{*}{$\begin{array}{c}\mathrm{Chi}^{2} \\
\chi^{2}=5.7\end{array}$} & \multirow{2}{*}{$0.017 *$} \\
\hline Female & $23(76.7 \%)$ & $14(46.7 \%)$ & & \\
\hline
\end{tabular}


Table 2: Prevalence of seven static malocclusions and results of the univariate analysis. $\left({ }^{*} p<0.05, * * P<0.005\right)$

\begin{tabular}{|c|c|c|c|c|}
\hline & Case group $(n=30)$ & Control group $(n=30)$ & Test & p-value \\
\hline Posterior cross bite & $5(16.70 \%)$ & $5(16.70 \%)$ & $\begin{array}{l}\mathrm{Chi}^{2} \\
\chi^{2=0}\end{array}$ & 1 \\
\hline Overjet $>7 \mathrm{~mm}$ & $0(0 \%)$ & $1(3.30 \%)$ & Fisher & 1 \\
\hline Overbite $>4 \mathrm{~mm}$ & $8(26.70 \%)$ & $2(6.70 \%)$ & $\begin{array}{c}\mathrm{Chi}^{2} \\
\chi^{2=} 4.32\end{array}$ & $0.038 *$ \\
\hline Anterior open bite & $1(3.30 \%)$ & $2(6.70 \%)$ & Fisher & 1 \\
\hline Crowding of the lower incisors & $7(23.30 \%)$ & $6(20.00 \%)$ & $\begin{array}{c}\mathrm{Chi}^{2} \\
\chi^{2=}=0.0982\end{array}$ & 0.754 \\
\hline Missing non-replaced posterior teeth & $7(23.30 \%)$ & $17(56.70 \%)$ & $\begin{array}{c}\mathrm{Chi}^{2} \\
\chi^{2=}=6.94\end{array}$ & $0.0084 * *$ \\
\hline
\end{tabular}

Table 3: Prevalence of four dynamic occlusal variables and results of the univariate analysis. $\left({ }^{*} p<0.05, * * P<0.005\right)$

\begin{tabular}{|c|c|c|c|c|}
\hline & Case group $(n=30)$ & Control group $(n=30)$ & Test & p-value \\
\hline Protrusion interference & $3 / 26(11.50 \%)$ & $8 / 29(27.60 \%)$ & $\begin{array}{c}\mathrm{Chi}^{2} \\
\chi^{2}=2.2\end{array}$ & 0.1374 \\
\hline Interference in laterotrusion & $21 / 29(72.40 \%)$ & $9(30.00 \%)$ & $\begin{array}{c}\text { Chi }^{2} \\
\chi^{2}=10.614\end{array}$ & $0.0011 * *$ \\
\hline Laterotrusive Interference & $14 / 29(48.30 \%)$ & $4(13.30 \%)$ & $\begin{array}{c}\mathrm{Chi}^{2} \\
\chi^{2}=8.49\end{array}$ & $0.0036 * *$ \\
\hline Mediotrusive Interference & $16 / 29(55.20 \%)$ & $7(23.30 \%)$ & $\begin{array}{c}\mathrm{Chi}^{2} \\
\chi^{2}=6.28\end{array}$ & $0.0122 *$ \\
\hline RCP contacts & $11(36.70 \%)$ & $9(30.00 \%)$ & $\begin{array}{c}\mathrm{Chi}^{2} \\
\chi^{2}=0.3\end{array}$ & 0.5839 \\
\hline Asymmetric RCP contacts & $8(26.70 \%)$ & $3(10.00 \%)$ & $\begin{array}{c}\mathrm{Chi}^{2} \\
\chi^{2}=2.78\end{array}$ & 0.0953 \\
\hline
\end{tabular}

Table 4: Multivariate analysis.

\begin{tabular}{|c|c|c|c|c|c|c|}
\hline \multicolumn{7}{|c|}{ Variables in the Equation } \\
\hline & & $\begin{array}{l}\mathrm{B} \text { (=Slope of the } \\
\text { regression line) }\end{array}$ & & & & \\
\hline \multirow{6}{*}{ Step $1^{\mathrm{a}}$} & Age & -0.047 & 0.035 & 1.802 & 1 & 0.179 \\
\hline & Gender (1) & 1.656 & 0.973 & 2.893 & 1 & 0.089 \\
\hline & Overbite >4 & 2.685 & 1.239 & 4.694 & 1 & 0.03 \\
\hline & $\begin{array}{l}\text { Interference in } \\
\text { laterotrusion }\end{array}$ & 4.093 & 1.134 & 13.029 & 1 & 0.0001 \\
\hline & $\begin{array}{l}\text { Protrusion } \\
\text { interference }\end{array}$ & -2.732 & 1.112 & 6.032 & 1 & 0.014 \\
\hline & Constant & -1.369 & 1.607 & 0.726 & 1 & 0.394 \\
\hline
\end{tabular}

${ }^{a}$ Variable(s) entered on step 1: Age, Gender, and Overbite $>4$, Interference in laterotrusion, Protusion Interference

Table 5: Prevalence of normocclusion criteria: bilateral class I and overbite $\leq 4 \mathrm{~mm}\left({ }^{*} \mathrm{p}<0.05, * * \mathrm{P}<0.005\right)$.

\begin{tabular}{|l|c|c|c|c|}
\hline & $\begin{array}{c}\text { Case group } \\
(\mathbf{n}=\mathbf{3 0})\end{array}$ & $\begin{array}{c}\text { Control group } \\
(\mathbf{n}=\mathbf{3 0})\end{array}$ & Test & p-value \\
\hline Bilateral Class I & $15(50.00 \%)$ & $21(70.00 \%)$ & $\begin{array}{c}\mathrm{Chi}^{2} \\
\chi^{2}=2.5\end{array}$ & 0.114 \\
\hline $\begin{array}{l}\text { Bilateral Class I AND } \\
\text { Overbite } \leq 4 \mathrm{~mm}\end{array}$ & $11(36.70 \%)$ & $20(66.70 \%)$ & $\begin{array}{c}\mathrm{Chi}^{2} \\
\chi^{2}=5.4\end{array}$ & $\mathbf{0 . 0 2 *}$ \\
\hline
\end{tabular}

have focused on dynamic malocclusions [1], yet they seem to be more associated with muscular TMD than other occlusion disorders [4].

In our study, statistical analysis of static and dynamic malocclusions showed that the number of patients with normocclusion was statistically more important in the control group than in the case group, and showed a statistically significant association between TMD and three malocclusions: overbite $>4 \mathrm{~mm}$, mediotrusive interferences, and laterotrusive interferences. These results are consistent with some results of literature. Evidence of a link between TMD and mediotrusive interferences or an important overbite was found in numerous studies [4,13-16]. On the contrary, other malocclusions significantly linked to TMD in literature were not significant in this study, like important retruded contact position (RCP) to Maximum Intercuspation (MI) slide length [13-17], absence of canine guidance [14,15], crossbite, overjet, and midline discrepancy [16].

These variations may be explained by differences in protocols: some studies were only interested in disk displacements, or only in muscular pains, others only included women, or only men.

Some authors investigated malocclusions according to clinical signs of TMD: Chiappe, et al. [14] showed that absence of canine guidance; mediotrusive interference and slide from RCP to $\mathrm{MI} \geq 2 \mathrm{~mm}$ are associated with disk displacement [14]. A study led by Ferreira et al. [5] showed that overjet has a statistically significant correlation with myofascial pain. However, this study did not show correlation between occlusal alterations or parafunctional habits and disk displacement. Fantoni, et al. [4] underlined the importance of differentiating forms of TMD to clarify their association with occlusion features. 
Table 6: Prevalence of oral parafunctions $\left({ }^{*} p<0.05, * * p<0.005\right)$.

\begin{tabular}{|c|c|c|c|c|}
\hline & Case group $(n=30)$ & Control group $(n=30)$ & Test & p-value \\
\hline \multicolumn{5}{|l|}{ Parafunctions } \\
\hline Nail biting & $13(43.3 \%)$ & $8(26.7 \%)$ & $\begin{array}{c}\text { Chi }^{2} \\
\chi^{2}=1.83\end{array}$ & 0.176 \\
\hline Lips and cheeks biting & $18(60.0 \%)$ & $8(26.7 \%)$ & $\begin{array}{c}\mathrm{Chi}^{2} \\
\chi^{2}=6.79\end{array}$ & $0.009 * *$ \\
\hline Penschewing & $7(23.3 \%)$ & $7(23.3 \%)$ & $\begin{array}{l}\mathrm{Chi}^{2} \\
\chi^{2}=0\end{array}$ & 1 \\
\hline Mandibular jaw moves habit & $13(43.3 \%)$ & $4(13.3 \%)$ & $\begin{array}{c}\mathrm{Chi}^{2} \\
\chi^{2}=6.65\end{array}$ & $0.0099 * *$ \\
\hline Teeth contact (between maxillar and mandibular teeth) & $17(56.7 \%)$ & $14(46.7 \%)$ & $\begin{array}{c}\text { Chi }^{2} \\
\chi^{2}=0.6\end{array}$ & 0.438 \\
\hline Teeth grinding & $14(46.7 \%)$ & $9(30.0 \%)$ & $\begin{array}{c}\text { Chi }^{2} \\
\chi^{2}=1.76\end{array}$ & 0.184 \\
\hline Teeth clenching & $21(70.0 \%)$ & $12(40.0 \%)$ & $\begin{array}{c}\text { Chi }^{2} \\
\chi^{2}=5.45\end{array}$ & $0.02 *$ \\
\hline Other parafunctions (described by patient) & $1(3.3 \%)$ & $0(0.0 \%)$ & Fisher & 1 \\
\hline
\end{tabular}

Table 7: Multivariate analysis.

\begin{tabular}{|c|c|c|c|c|c|c|}
\hline \multicolumn{7}{|c|}{ Variables in the Equation } \\
\hline & & B & S.E. & Wald & df & Sig. \\
\hline \multirow{8}{*}{ Step $1^{a}$} & Age & -0.011 & 0.029 & 0.154 & 1 & 0.695 \\
\hline & Gender(1) & 0.938 & 0.805 & 1.357 & 1 & 0.244 \\
\hline & Overbite>4 & 2.697 & 1.23 & 4.807 & 1 & 0.028 \\
\hline & Interference in laterotrusion & 2.945 & 0.937 & 9.869 & 1 & 0.002 \\
\hline & Onychophagia & 1.113 & 0.788 & 1.999 & 1 & 0.157 \\
\hline & Mandible movement & 1.624 & 0.929 & 3.054 & 1 & 0.081 \\
\hline & Teeth grinding & 0.865 & 0.831 & 1.083 & 1 & 0.298 \\
\hline & Constant & -3.434 & 1.745 & 3.872 & 1 & 0.049 \\
\hline
\end{tabular}

${ }^{a}$ Variable(s) entered on step 1: Age; Gender; Overbite>4, Interference in laterotrusion, Onychophagia, Mandible movement, Teeth grinding.

Oral parafunctions are considered as a risk factor [8]. In this study, there was a statistically significant difference between patients with and without TMD in three parafunctions: teeth clenching, lips and cheeks biting, mandibular jaw moves habits.

Almost all patients of the two groups ( 30 case patients and 28 control patients) had one parafunction, but it was the association of several parafunctions that was pathogenic for temporomandibular joint. This result demonstrated parafunctions role in TMD appearance, as some studies have already shown $[5,8]$.

Some studies did not show the development of TMD symptoms when creating interferences in healthy patients, but a possible aggravation of muscular pain in individuals with high level of parafunctions compared to individuals with a low frequency of parafunctions $[1,8,18]$.

Even taking into account the limits of our study, it demonstrated with good confidence the role of two etiologies in TMD development: some occlusal factors and oral parafunctions. However, research on the etiology of TMD will need to be continued. Since the main limitation of our study was sample size, this work will have to be carried on with larger samples, in order to highlight possible differences according to gender and age. In addition, a future study could investigate the role of iatrogenic malocclusions in the development of TMD, compared to natural malocclusions.

Some authors have highlighted the efficiency of treatments such as selective grinding in the correction of occlusal problems and TMDs $[19,20]$. However, the American Association of Dental Research advises first a non-invasive and reversible treatment [21]. Some authors agree to say that irreversible occlusal treatments have to be banned, non-invasive treatments having good results [12,22].

Therefore, a behavioral re-education to reduce parafunctional activity could be the first phase of the care for TMD patients and could prevent TMD development. Besides, to prevent TMD development, normocclusion criteria must be respected in dental care.

\section{Acknowledgments}

The authors thank Céline Allaire for editorial assistance, and Catherine Rouxel for clinical assistance. 


\section{References}

1. Manfredini D, Perinetti G, Stellini E, Di Leonardo B, Guarda-Nardini $L$ (2015) Prevalence of static and dynamic dental malocclusion features in subgroups of temporomandibular disorder patients: Implications for the epidemiology of the TMD-occlusion association. Quintessence Int 46: 341-349.

2. Barrera-Mora JM, Espinar Escalona E, Abalos Labruzzi C, Llamas Carrera JM, Ballesteros EJ, et al. (2012) The relationship between malocclusion, benign joint hypermobility syndrome, condylar position and TMD symptoms. Cranio 30: 121-130.

3. Xie Q, Li X, Xu X (2013) The difficult relationship between occlusal interferences and temporomandibular disorder-insights from animal and human experimental studies. J Oral Rehabil 40: 279-295.

4. Fantoni F, Chiappe G, Landi N, Romagnoli M, Bosco M (2010) A stepwise multiple regression model to assess the odds ratio between myofascial pain and 13 occlusal features in 238 Italian women. Quintessence Int 41: e54-e61.

5. Ferreira FM, Simamoto-Junior PC, Novais VR, Tavares M, FernandesNeto AJ (2014) Correlation between temporomandibular disorders, occlusal factors and oral parafunction in undergraduate students. Braz J Oral Sci 13: 281-287.

6. Orthlieb JD, Ré JP, Jeany M, Giraudeau A (2016) Temporomandibular joint, occlusion and bruxism. Rev Stomatol Chir Maxillofac Chir Orale 117: 207-211.

7. Manfredini D, Bucci MB, Montagna F, Guarda-Nardini L (2011) Temporomandibular disorders assessment: medicolegal considerations in the evidence-based era. J Oral Rehabil 38: 101119.

8. Michelotti A, Cioffi I, Landino D, Galeone C, Farella M (2012) Effects of experimental occlusal interferences in individuals reporting different levels of wake-time parafunctions. J Orofac Pain 26: 168-175.

9. Manfredini D, Perinetti G, Guarda-Nardini L (2014) Dental malocclusion is not related to temporomandibular joint clicking: a logistic regression analysis in a patient population. Angle Orthod 84 310-315.

10. Badel T, MarottiM, Pavicin I, Basić-Kes V (2012) Temporomandibular disorders and occlusion. Acta Clin Croat 51: 419-424.

11. Türp JC, Schindler H (2012) The dental occlusion as a suspected cause for TMDs: epidemiological and etiological considerations. J Oral Rehabil 39: 502-512.
12. Manfredini D, Castroflorio T, Perinetti G, Guarda-Nardini L (2012) Dental occlusion, body posture and temporomandibular disorders: where we are now and where we are heading for. J Oral Rehabil 39: 463-471.

13. Landi N, Manfredini D, Tognini F, Romagnoli M, Bosco M (2004) Quantification of the relative risk of multiple occlusal variables for muscle disorders of the stomatognathic system. J Prosthet Dent 92: 190-195.

14. Chiappe G, Fantoni F, Landi N, Biondi K, Bosco M (2009) Clinical value of 12 occlusal features for the prediction of disc displacement with reduction (RDC/TMD Axis I group Ila). J Oral Rehabil 36: 322-329.

15. Haralur SB (2013) Digital evaluation of functional occlusion parameters and their association with temporomandibular disorders. J Clin Diagn Res 7: 1772-1775.

16. Pullinger AG, Seligman DA (2000) Quantification and validation of predictive values of occlusal variables in temporomandibular disorders using a multifactorial analysis. J Prosthet Dent 83: 66-75.

17. Badel T, Marotti M, Krolo I, Kern J, KerosJ (2008) Occlusion in patients with temporomandibular joint anterior disk displacement. Acta Clin Croat 47: 129-136.

18. Michelotti A, Farella M, Steenks MH, Gallo LM, Palla S (2006) No effect of experimental occlusal interferences on pressure pain thresholds of the masseter and temporalis muscles in healthy women. Eur J Oral Sci 114: 167-170.

19. Fernández-González FJ, Cabero-López J, Brizuela A, Suazo I, PérezPevida E, et al. (2017) Efficacy of Selective Grinding Guided by an Occlusal Splint in Management of Myofascial Pain: A Prospective Clinical Trial. Open Dent J 11: 301-311.

20. Ferreira CF, Prado AM, Pereira MA, Cardoso AC (2016) The Value of Occlusion in Dentistry: A Clinical Report Showing the Correction of an Anterior Reverse Articulation with Selective Occlusal Adjustment. J Prosthodont 25: 407-410.

21. Greene CS, Klasser GD, Epstein JB (2010) Revision of the American Association of Dental Research's Science information statement about Temporomandibular Disorders. J Can Dent Assoc 76: a115.

22. Luther F (2007) TMD and occlusion part II. Damned if we don't? Functional occlusal problems: TMD epidemiology in a wider context. Br Dent J 202: 38-39. 